\title{
BSG2017
}

Endoscopy (including video)*

\section{BSG2017-925 \\ ASSESSMENT OF TRAINING NEEDS AND DEVELOPMENT OF A SIMULATION BASED TRAINING PROGRAMME FOR SEMI-AUTOMATED ROBOTIC COLONOSCOPY.}

Maja Kopczynska*, Stephanie Smits, Rebecca Hopps, Neil Warren, Stuart Goddard, Xujiong Ye, Sunil Dolwani

Introduction: Early diagnosis of colorectal cancer results in better patient outcomes. Reported barriers to screening amongst nonresponders, delays in diagnostic pathways, and current constraints in colonoscopy capacity contribute to delays in diagnosis. We hypothesise that changing the diagnostic paradigm through primary care based semi-automated robotic colonoscopy may improve access and engagement. This project involved development of a training programme with a simulation training model to understand the specific training needs and methods of fulfilling these for the potential ultimate users of primary care based robotic colonoscopy.

Method: Participants with varying degree of skills and background knowledge in colonoscopy enrolled included 3 expert endoscopists, 3 trainee endoscopists, 2 novices and 2 experienced video gamers. All participants performed colonoscopies on a validated 'colonoscopy suitcase' model developed at Welsh Institute of Minimal Access Therapy. Before testing each participant received both verbal and written instructions on the study and information about the semi-automated robotic endoscope along with a familiarisation period with the device. Quantitative parameters recorded related to procedure times and lesion detection during the procedure. Operators filled out a questionnaire evaluating the robotic colonoscope. Some operators participated in a follow up session to analyse the learning curve of robotic endoscopy.

Results: On average, experts took the shortest time to reach the caecum, followed by video gamers, trainees then novices. Polyp detection rate (proportion of total number in the model colon) was the highest in the novice group (91.67\%) followed by the experts $(86.11 \%)$, then equally, trainees and video gamers (79.17\%). 4 out of 9 participants attended the second session where they repeated the procedure from the first session. Each participant had a lower caecal intubation time during session 2 in comparison with session 1, with range of improvement between $30 \%$ and $70 \%$. Each of the participants also had the same or higher polyp detection rate with range of improvement between $0 \%$ and $25 \%$. Qualitative assessment of feedback from all participants indicated that most operators felt that the role of the novel test was likely to be as a diagnostic procedure in an out of hospital setting. Expert operators felt that training in the device was easier but also provided less ability to torque steer due to automated sequences.

Conclusion: This project is the first step in the assessment of specific training needs and development of a training programme in robotic endoscopy. This study also shows a potential to reduce the length of time for skills acquisition associated with standard colonoscopy training through the use of semi-automated robotic devices.

Disclosure of Interest: None Declared

Keywords: colonoscopy, COLORECTAL CANCER, early diagnosis, robotic, technology, training 